\title{
Feynman Formulas Representation of Semigroups Generated by Parabolic Difference-Differential Equations
}

\author{
V. Sakbaev, A. Yaakbarieh \\ Moscow Institute of Physics and Technology, People's Friendship University of Russia, Moscow, Russia \\ Email: fumi2003@mail.ru, amirmath20@yahoo.com
}

Received July 10, 2012; revised October 27, 2012; accepted November 1, 2012

\begin{abstract}
We establish that the Laplas operator with perturbation by symmetrised linear hall of displacement argument operators is the generator of unitary group in the Hilbert space of square integrable functions. The representation of semigroup of Cauchy problem solutions for considered functional differential equation is given by the Feynman formulas.
\end{abstract}

Keywords: Difference-Differential Equations; Semigroup; Feynman Formula; Chernoff Theorem

\section{Introduction}

In this paper we investigate the questions of correct resolvability of Cauchy problem for modeling parabolic differential-difference the equations of the form

$$
\begin{aligned}
& u_{t}(x, t)=\Delta u(x, t) \\
& +\sum_{i=1}^{N} a_{k}\left(u\left(x-h_{k}, t\right)+u\left(x+h_{k}, t\right)\right),(x, t) \in R \times R_{+},
\end{aligned}
$$

supplemented with the initial data

$$
u(x, 0)=u_{0}(x), x \in R .
$$

Here $N \in \mathbf{N}, a_{k} \in R, h_{k} \geq 0$ for any $k \in 1, \cdots, N, u_{0}$ is a given function and $\Delta=\partial^{2} \partial x^{2}$ is self-adjoint Laplas operator in the space $L_{2}(R)$ with domain $W_{2}^{2}(R)$. The equations of this form arise at the description of the phenomena of diffusion or heat conductivity with the sources, nonlocally dependent on the state $u$. Physically the state $u$ means the density distribution of the concentration or the temperature. In particular, the equations of a kind (1) arise at research of problems of management by the phenomena of a heat transfer in which dynamics of a state $u$ is given by the differential equation

$$
u_{t}(x, t)=\Delta u(x, t)+g(t, x, u),
$$

with management $g$. We obtain the Equation (1) in case when management $g$ is given by the action on a state function $u$ of a deviation argument operators in a composition with operators of differentiation and multiplication by the function (see [1-3]).

In this work we obtain the representation of semigroup solutions of the Cauchy problem for the functionaldifferential equation through the Feynman formula (see [4]). It means that although the representation of the evolutionary operator of the Cauchy problem (1) can be defined only in terms of the spectral decomposition (in the simplest situation in terms of the Fourier transform of the solution), nevertheless we obtain an approximation of the evolutionary operator by sequence of $n$-fold compositions of integrated operators which kernels are elementary functions.

In the terms of the monography [1], differentialdifference Equation (1) concerns to type mixed differential-difference equation without a deviation on time. Nonlinear parabolic differential-difference the equations arise in the investigation of nonlinear optical systems with a feedback (see [3]). In work [5] the mixed problem for nonlinear parabolic differential-difference equations had been formulated. Also it was established what properties distinguish the specified problem from the mixed problem for parabolic differential equations. The linear Cauchy problem (1), (2) can be considered as a linearization of specified nonlinear mixed problems.

Firstly we prove the correctness of Cauchy problem (1), (2) by using of Fourier transformation and obtain the representation of Fourier image of its solution. After that we construct the approximation of solution by Feynman formulas. We extend the obtained result onto the operators with distributed deviation of the space argument.

The obtained results gives not only the expression of the decision of Cauchy problem with the help of constructive algorithms, but also the investigation of probabilistic structure of the phenomena reject the arguments in the heat equation. The above Feynman formulas define the approximation of a Markov random process, such that the mean value of some functional on this process is the solutions of the Cauchy problem. 


\section{Correct Resolvability of the Cauchy Problem and Generation of Semigroup Operators}

Let us determine the solution of the Equation (1), satisfying the initial condition (2).

Definition. A strong solution of the Cauchy problem (1), (2) call the function

$u \in C\left([0,+\infty), W_{2}^{2}(R)\right) \cap C^{1}\left((0,+\infty), L_{2}(R)\right)$ which Satisfies the equation (1) and condition (2).

Function $u \in C\left([0,+\infty), L_{2}(R)\right)$ call a weak solution of the Cauchy problem (1), (2), if there is a sequence of initial data $\left\{u_{0 k}\right\}$ such that

1) the sequence $\left\{u_{0 k}\right\}$ converges in space $H$ to the element $u_{0}$,

2) for each $k \in \mathbf{N}$ there is a strong solution $u_{k}$ of the Cauchy problem with initial condition $u_{0 k}$,

$3)$ the sequence of functions $\left\{u_{k}\right\}$ converges to functions $u$ in space $C\left([0,+\infty), L_{2}(R)\right)$.

Note that both strong and weak solution satisfies the Equation (1) and condition (2) in the sense of the integral identity.

Suppose that the existence of solution $u(x, t), t \geq 0, x \in R$, of Cauchy problem (1), (2) is obtained. To find a representation of the solution of the Cauchy problem (1), (2) through the initial condition, we apply the Fourier transform $F$ to the left and right part of Equation (1):

$$
\begin{aligned}
& F\left\{u_{t}(x, t)\right\}=F\{\Delta u(x, t)\} \\
& +\sum_{i=1}^{N} F\left\{a_{k}\left(u\left(x-h_{k}, t\right)+u\left(x+h_{k}, t\right)\right)\right\},
\end{aligned}
$$

Let the function $U(s, t), s \in R, t \in(0,+\infty)$ be the Fourier transform $u(x, t)$ in the first variable. Then Equation (3) takes the form:

$$
\begin{aligned}
& U_{t}(s, t)=-s^{2} U(s, t) \\
& +\sum_{i=1}^{N} a_{k}\left(U(s, t) \exp \left(i s h_{k}\right)+U(s, t) \exp \left(-\mathrm{i} s h_{k}\right)\right)^{\prime}
\end{aligned}
$$

and the initial condition (2) transforms into the equation:

$$
U(s, 0)=U_{0}(s) \equiv F\left(u_{0}\right)(s), s \in R .
$$

Then by using of the equations

$$
\begin{aligned}
& U_{t}(s, t)=-s^{2} U(s, t) \\
& +\sum_{i=1}^{N} a_{k}\left(\exp \left(i s h_{k}\right)+\exp \left(-\mathrm{i} s h_{k}\right)\right) U(s, t)
\end{aligned}
$$

and the initial condition we obtain that

$$
U(s, t)=U_{0}(s) \exp \left[\left(-s^{2}+2 \sum_{i=1}^{N} a_{k} \cos \left(s h_{k}\right)\right) t\right] .
$$

Thus, established the following statements
Proposition 1. If the Cauchy problem (1), (2) has a solution $u$, then the Fourier transform $U(s, t)$ defined by equality (4).

Theorem 1. The formula (4) defines a strongly continuous semigroup $U(t), t>0$, transformation of the space $L_{2}(R)$.

In fact, according to the unitarity of the the Fourier transform in space $H=L_{2}(R)$ it is sufficient to verify, that the one-parametrical family of operators $\tilde{U}(t), t>0$, of multiplication on the function $U(s, t), t \in R_{+}$, is strongly continuous semigroup operators in space $L_{2}(R)$ with norm not greater than one. Semigroup property $U\left(s, t_{1}\right) U\left(s, t_{2}\right)=U\left(s, t_{1}+t_{2}\right)$ follows from the properties of the exponential function. The strong continuity in point $t=0$ of operator-function $\tilde{U}(t), t \geq 0$ follows from the uniform on any interval of real line convergence of function $U(s, t), t \in R_{+}, s \in R$, to the unit function $1(s), s \in R$, at $t \rightarrow 0$. Then the strong continuity at any point $t>0$ follows from the semigroup property. Moreovet the type of above semigroupe $\omega$ is equal to the value $\sup f(s)$, where $f(s)=-s^{2}+2 \sum_{i=1}^{N} a_{k} \cos \left(s h_{k}\right), s \in R$. Therefore $\omega \leq 2 \sum_{k=1}^{N}\left|a_{k}\right|$. The theorem 1 is proved.

Theorem 2. The Cauchy problem (1), (2) has a unique generalized solution $u(t), t \geq 0$, which is defined as the action of the semigroup $\mathrm{U}(t), t \geq 0$ on the initial condition $u_{0}$.

In fact, according to the proposition 1 , if the solution of the Cauchy problem (1), (2) exists, then it representable in the form

$$
\begin{aligned}
& u(x, t)=u_{0}(x) \\
& * F^{-1}\left\{\exp \left[\left(-s^{2}+2 \sum_{i=1}^{N} a_{k} \cos \left(s h_{k}\right)\right) t\right]\right\} .
\end{aligned}
$$

Conversely, if function $U(s, t), s \in R, t \geq 0$, is defined by the equality (4) then the function

$u(x, t)=F^{-1}\{U(s, t)\}$ satisfy the equality (5), the equality (1) and the condition (2). In fact, if the function $U_{0}(s) \in L_{2}(R)$ satisfy the condition $s^{2} U_{0}(s) \in L_{2}(R)$ then the function $U(s, t)$ (see (4)):

belongs to space

$$
C\left([0,+\infty), L_{2}(R)\right) \cap C^{1}\left((0,+\infty), L_{2}(R)\right) ;
$$

satisfies the inclusion $s^{2} U(s, t) \in C\left([0,+\infty), L_{2}(R)\right)$ and the equation

$$
\frac{\partial}{\partial t} U(s, t)=\left(-s^{2}+2 \sum_{i=1}^{N} b_{k} \cos \left(s h_{k}\right)\right) U(s, t) .
$$

Then, by the unitarity of the Fourier transform, for each initial function $u_{0} \in W_{2}^{2}(R)$ there is the function (5) which is a strong solution of the Cauchy problem (1), (2). Hence, the formula (5) defines the image of the 
function $u_{0} \in W_{2}^{2}(R)$ under the action of the semigroup operators $\boldsymbol{U}(t), t \geq 0$ According to Theorem 1 the semigroup $\boldsymbol{U}(t)$ has a bounded exponential growth $\omega$, then the semigroup $\boldsymbol{U}(t)$ supposes continuation by continuity from space $W_{2}^{2}(R)$ onto the space $L_{2}(R)$. Hence for any initial condition $u_{0} \in L_{2}(R)$ the function $u(t)=\boldsymbol{U}(t) u_{0}$, is a generalized solution of the Cauchy problem (1), (2). Theorem 2 is proved.

Corollary 1. The generator of the semigroup

$\boldsymbol{U}(t), t \geq 0$ is operator $\boldsymbol{L}=\Delta+\sum_{k=1}^{N} a_{k}\left(\boldsymbol{S}_{h_{k}}+\boldsymbol{S}_{-h_{k}}\right)$,

where $\boldsymbol{S}_{h}$ is the shift operator on value $h \in R$.

The obtained representation of the solution of the Cauchy problem (1), (2) is not constructive. For approximation of the solution obtained by using of sequences of multiple integrals of elementary functions we use the approach from papers $[4,6,7]$ based on the Feynman formulas.

\section{Chernoff's Approximation of Solutions of the Cauchy Problem}

Following the approach offered in [4], we define the operator-valued function equivalent in Chernoff sense to the semigroup of operators $\boldsymbol{U}(t), t>0$. Chernoff's theorem (see [8]) states that:

Let the operator-valued function $\boldsymbol{F}(t), t \geq 0$, with values in Banach space $B(H)$ continuous in strong operational topology, supposes the estimation

$\|\boldsymbol{F}(t)\|_{B(H)} \leq 1+\alpha t, t \geq 0$, for some $\alpha>0$ and, moreover, operator $\boldsymbol{F}^{\prime}(0)$ is closable and its closure is the generator of a strongly continuous semigroup $\boldsymbol{U}(t), t>0$. Then for any $u \in H$ and any $T>0$ the equality $\lim _{n \rightarrow \infty} \sup _{t \in[0, T]}\left\|\boldsymbol{U}(t) u-(\boldsymbol{F}(t n))^{n} u\right\|_{H}=0$.

By following the definition in [4] the operator-valued function $\boldsymbol{F}(t), t \geq 0$, will be called equivalent by Chernoff semigroup $\boldsymbol{U}(t), t \geq 0$. if for any $u \in H$ the equality

$$
\limsup _{n \rightarrow \infty[0, T]}\left\|(\boldsymbol{F}(t n))^{n} u-\boldsymbol{U}(t) u\right\|_{H}=0
$$

satisfies for all $T>0$.

Let us assume that $N=1$. For given in the Equation (1) parameters $a_{1}=a$ and $H_{1}=h$ we consider the operator-valued function $\boldsymbol{F}_{a, h}: R_{+} \rightarrow B(H)$, defined on $R_{+}=[0,+\infty)$ and taking values in a Banach space

$B(H)$ of bounded linear operators in Hilbert space $H$. For each value of $t \geq 0$ we define its value $\boldsymbol{F}_{a, h}(t)$ by the equality

$$
\begin{aligned}
& \boldsymbol{F}_{a, h}(t) u_{0}=u(t, x) \\
& =\frac{1}{\sqrt{2 \pi t}} \int_{-\infty}^{+\infty}\left[\exp \left(\frac{-(x-y)^{2}}{4 t}\right)\right. \\
& \left.+a t\left(\exp \left(\frac{-(x-y-h)^{2}}{4 t}\right)+\exp \left(\frac{-(x-y+h)^{2}}{4 t}\right)\right)\right] u_{0}(y) \mathrm{d} y
\end{aligned}
$$

which is satisfied for arbitrary function $u_{0}$ from the dense in space $H$ linear manifold $D=C_{0}^{\infty}(R)$. The proposed form of Chernoff's approximation of the semigroup related to the fact that the first term in formula (6) corresponds to the dynamics, generated by the unperturbed heat equation, and the second and third terms for small values of the variable $t$ presente the influence of the displaced sources.

We verify the conditions of Chernoff's theorem for operator-valued function $\boldsymbol{F}_{a, h}(t), t \in R_{+}$.

Lemma 1. If $u_{0} \in D$ the equality holds

$$
\begin{aligned}
& \left.\frac{\mathrm{d}}{\mathrm{d} t} \boldsymbol{F}_{a, h}(t) u_{0}\right|_{t=0} \\
& =\frac{\partial^{2} u_{0}}{\partial x^{2}}(x)+a\left(u_{0}(x+h)+u_{0}(x-h)\right) .
\end{aligned}
$$

To prove of lemma 1 we compute the function $\left(\frac{\partial u}{\partial t}-\frac{\partial^{2} u}{\partial x^{2}}\right)(t, x), t>0, x \in R$. Since

$$
\begin{aligned}
\left(\frac{\partial u}{\partial t}\right)= & \frac{-\pi}{2 \pi t \sqrt{2 \pi t}} \int_{-\infty}^{+\infty}\left[\exp \left(\frac{-(x-y)^{2}}{4 t}\right)+a t\left(\exp \left(\frac{-(x-y-h)^{2}}{4 t}\right)+\exp \left(\frac{-(x-y+h)^{2}}{4 t}\right)\right)\right] u_{0}(y) \mathrm{d} y \\
& +\frac{1}{\sqrt{2 \pi t}} \int_{-\infty}^{+\infty}\left[\frac{(x-y)^{2}}{4 t^{2}} \exp \left(\frac{-(x-y)^{2}}{4 t}\right)+\left(\exp \left(\frac{-(x-y-h)^{2}}{4 t}\right)+\exp \left(\frac{-(x-y+h)^{2}}{4 t}\right)\right)\right. \\
& \left.+a t\left(\frac{(x-y-h)^{2}}{4 t^{2}} \exp \left(\frac{-(x-y-h)^{2}}{4 t}\right)+\frac{(x-y+h)^{2}}{4 t^{2}} \exp \left(\frac{-(x-y+h)^{2}}{4 t}\right)\right)\right] u_{0}(y) \mathrm{d} y
\end{aligned}
$$

and 


$$
\begin{aligned}
\frac{\partial^{2} u}{\partial x^{2}}= & \frac{1}{\sqrt{2 \pi t}} \int_{-\infty}^{+\infty}\left[\frac{-1}{2 t} \exp \left(\frac{-(x-y)^{2}}{4 t}\right)+\frac{(x-y)^{2}}{4 t^{2}} \exp \left(\frac{-(x-y)^{2}}{4 t}\right)\right. \\
& +a t\left(\frac{-1}{2 t} \exp \left(\frac{-(x-y-h)}{4 t}\right)+\frac{(x-y-h)^{2}}{4 t^{2}} \exp \left(\frac{-(x-y-h)}{4 t}\right)\right. \\
& \left.\left.-\frac{1}{2 t} \exp \left(\frac{-(x-y+h)^{2}}{4 t}\right)+\frac{(x-y+h)^{2}}{4 t^{2}} \exp \left(\frac{-(x-y+h)^{2}}{4 t}\right)\right)\right] u_{0}(y) \mathrm{d} y
\end{aligned}
$$

then

$$
\left(\frac{\partial u}{\partial t}-\frac{\partial^{2} u}{\partial x^{2}}\right)(t, x)=\frac{1}{\sqrt{2 \pi t}} \int_{-\infty}^{+\infty}\left(a \exp \frac{-(x-y-h)^{2}}{4 t}+a \exp \frac{-(x-y+h)^{2}}{4 t}\right) u_{0}(y) \mathrm{d} y .
$$

Consequently

$$
\begin{aligned}
& \left.\frac{\mathrm{d}}{\mathrm{d} t} \boldsymbol{F}_{a, h}(t) u_{0}\right|_{t=0} \\
& =\lim _{t \rightarrow 0}\left[\frac{\partial^{2} u}{\partial x^{2}}(t, x)+\frac{a}{\sqrt{2 \pi t}} \int_{-\infty}^{+\infty}\left(\exp \frac{-(x-y-h)^{2}}{4 t}+\exp \frac{-(x-y+h)^{2}}{4 t}\right) u_{0}(y) \mathrm{d} y\right] \\
& =\frac{\partial^{2} u_{0}}{\partial x^{2}}(x)+a\left(u_{0}(x+h)+u_{0}(x-h)\right) .
\end{aligned}
$$

Lemma 1 is proved.

For arbitrary $N \in \mathbf{N}$ and for given in Equation (1) coefficients $a_{k}, h_{k}, k \in \overline{1, N}$ we define the operator function $\boldsymbol{F}_{A, H}(t), t \geq 0$, assuming that for each function $u_{0} \in D$ its image $\boldsymbol{F}_{A, H}(t) u_{0}$ defined by equality

$$
\begin{aligned}
& \boldsymbol{F}_{A, H}(t) u(x) \\
& =\frac{1}{\sqrt{2 \pi t}} \int_{-\infty}^{+\infty}\left[\exp \left(\frac{-(x-y)^{2}}{4 t}\right)\right. \\
& \left.+\sum_{k=1}^{N} a_{k} t\left(\exp \left(\frac{-\left(x-y-h_{k}\right)^{2}}{4 t}\right)+\exp \left(\frac{-\left(x-y+h_{k}\right)^{2}}{4 t}\right)\right)\right] .
\end{aligned}
$$

Hence, by lemma 1 on linear manifold $D$ the operator $\left.\frac{\mathrm{d}}{\mathrm{d} t} \boldsymbol{F}_{A, H}(t)\right|_{t=0}$ coincides with the generator of the semigroup $U(t), t>0$.

Lemma 2. The operator-valued function

$\boldsymbol{F}_{A, H}(t), t \geq 0$, is continuous in the strong operator topology on the semiaxis $t \geq 0$ and supposes an estimation on norm $\left\|\boldsymbol{F}_{a, h}(t)\right\|_{B(H)} \leq 1+2 t \sum_{k=1}^{N} a_{k}, t \geq 0$.

Firstly we prove this statement for the case $N=1$. The operator-valued function $\boldsymbol{F}_{a, h}(t)$ is the sum of function $\boldsymbol{F}_{0}(t)$ with the integral kernel of the heat equation and the function $\Phi_{a, h}(t)$ with the integral kernel

$$
\begin{aligned}
& \Phi_{a, h}(t, x, y)=\frac{1}{\sqrt{2 \pi t}} \\
& \cdot \int_{-\infty}^{+\infty}\left[a t\left(\exp \left(\frac{-(x-y-h)^{2}}{4 t}\right)+\exp \left(\frac{-(x-y+h)^{2}}{4 t}\right)\right)\right]
\end{aligned}
$$

And $\Phi_{a, h}(t)=a t F_{0}(t)\left(\mathbf{S}_{h}+\mathbf{S}_{-h}\right)$, where $\boldsymbol{S}_{ \pm h}$ - shift operators on the value of $\pm h$. It is well known that the operator-valued function $\boldsymbol{F}_{0}(t), t>0$, is continuous in the strong operational topology and uniformly bounded the norm topology: $\left\|\boldsymbol{F}_{0}(t)\right\|_{B(H)} \leq 1 \quad \forall t \geq 0$. The operators $\boldsymbol{S}_{ \pm h}$ are bounded and have unit normed. Hence, the operator-valued function $\Phi_{a, h}(t)$ is continuous in the strong operator topology and satisfy the estimate $\|\Phi\|_{B(H)} \leq 2|a| t$.

If $N \in \mathbf{N}$ then

$$
\boldsymbol{F}_{A, H}(t)=\boldsymbol{F}_{0}(t)+\sum_{k=1}^{N} a_{k} t \boldsymbol{F}_{0}(t)\left(\boldsymbol{S}_{h_{k}}+\boldsymbol{S}_{-h_{k}}\right), t \geq 0 .
$$

Therefore the operator-valued function $\boldsymbol{F}_{A, H}(t)$ is continuous in the strong operator topology and satisfy the estimate $\left\|\boldsymbol{F}_{A, H}(t)\right\|_{B(H)} \leq 1+2 t \sum_{k=1}^{N}\left|a_{k}\right|$ for any $t \geq 0$.

Thus lemma 2 is proved.

Lemmas 1 and 2 exibit that the function $\boldsymbol{F}_{A, H}(t)$, $t \geq 0$ satisfies all conditions of Chernoff theorem. Therefore the next theorem is proved as the main result 
of Feynman type representation of solution of Cauchy problem for functional differential equation with deviation of space argument.

Theorem 3. Let the above assumption on the parameters of equation (1) are satisfies. Then the operatorvalued function $\boldsymbol{F}_{A, H}(t), t \geq 0$, is equivalent on Chernoff semigroup $\boldsymbol{U}(t), t>0$.

\section{Some Generalization on the Case of Distributed Deviation of the Space Argument}

At the end of our article we study the Cauchy problem for the perturbed Equation (1), in which the deviation of the argument presented by the convolution of unknown function with some kernel. As such a perturbation we consider the equation

$$
\begin{aligned}
& u_{t}(x, t)=\Delta u(x, t) \\
& +\sum_{k=1}^{N} a_{k}\left(u\left(t-h_{k}\right)+u\left(t+h_{k}\right)\right) \\
& +\int_{R} K(x-y) u(y, t) \mathrm{d} y \\
& \equiv \boldsymbol{L}_{K} u(t, x), \quad(x, t) \in R \times R_{+},
\end{aligned}
$$

where $K$ is some even function of space $L_{1}(R)$ and the remaining terms are defined in the consideration of the Equation (1). Hence Fourier transform $\hat{K}$ of the function $K$ is a bounded continuous real-valued function.

Let us define $U(s, t)=\mathcal{F}\{u(x, t)\}$ and $\hat{K}=\mathcal{F}\{K\}$. According to our assumption $\hat{K} \in L_{\infty}(R)$. Then we obtaine the relation

$$
U_{t}(s, t)=\left[-s^{2}+\sum_{i=k}^{N} a_{k}\left(\exp \left(i s h_{k}\right)+\exp \left(-\mathrm{ish} h_{k}\right)\right)+\sqrt{2 \pi} \hat{K}(s)\right] U(s, t)
$$

by applying Fourier transform $F$ to the equality (8). Hence we obtain, that

$$
\begin{aligned}
U(s, t) & =\exp \left[\left(-s^{2}+2 \sum_{i=1}^{N} a_{k} \cos \left(s h_{k}\right)\right) t+\sqrt{2 \pi} t \hat{K}(s)\right] U_{0}(s) \\
& \equiv\left(\boldsymbol{U}_{K}(t) U_{0}\right)(s)
\end{aligned}
$$

and therefore

$$
u(x, t)=u(x) * \mathcal{F}^{-1}\left\{\exp \left[t\left(-s^{2}+2 \sum_{i=1}^{N} a_{k} \cos \left(s h_{k}\right)\right)\right] \exp [\sqrt{2 \pi} t \hat{K}(s)]\right\}
$$

Therefore the following analogue of Theorems 1 and 2:

Proposition 2. If the kernel $K \in L_{1}(R)$, then equality (9) defines a one-parameter semigroup of $\boldsymbol{U}_{K}(t), t \geq 0$, contractive transformations of the space $H$, and for any $u_{0} \in H$ of the Cauchy problem for Equation (8) has a unique solution defined by equality (10), i.e. by the action of the semigroup $U_{K}(t), t \geq 0$, on the initial condition $u_{0}$.

So that, to find the approximate solution of the Cauchy problem by Feynman formula, we define, generalizing the formula (6), operator-valued function

$\boldsymbol{F}_{A, H, K}(t), t \geq 0$, such that (see (7))

$$
\begin{aligned}
& \boldsymbol{F}_{A, H, K}(t) u_{0}=\boldsymbol{F}_{A, H}(t) u_{0} \\
& +\frac{1}{\sqrt{2 \pi t}} \int_{R} \exp \left(\frac{-(x-z)^{2}}{4 t}\right) K(z-y) \mathrm{d} z u_{0}(y) \mathrm{d} y
\end{aligned}
$$

Lemma 3. For any $u_{0} \in C_{0}^{\infty}(R)$ the equality $\left.\left(\frac{\mathrm{d}}{\mathrm{d} t} \boldsymbol{F}_{A, H, K}(t) u_{0}\right)\right|_{t=0}=\boldsymbol{L}_{K} u_{0}$ holds.

According to the formula (11)

$$
\begin{aligned}
& \left.\left(\frac{\mathrm{d}}{\mathrm{d} t} \mathcal{F}\left(\boldsymbol{F}_{A, H, K}(t) u_{0}\right)(s)\right)\right|_{t=0} \\
& =\left[-s^{2}+2 \sum_{k=1}^{N} a_{k} \cos \left(h_{k} s\right)+\hat{K}(s)\right] u(s)
\end{aligned}
$$

therefore $\left.\left(\frac{\mathrm{d}}{\mathrm{d} t} \boldsymbol{F}_{A, H, K}(t) u_{0}(x)\right)\right|_{t=0}=\boldsymbol{L}_{K} u_{0}$, hence lemma 3 is proved.

Lemma 4. Operator-valued function $\boldsymbol{F}_{A, H, K}(t), t \geq 0$, is continuous in the strong operator topology on the $t \geq 0$, and admits the estimate in the norm

$$
\left\|\boldsymbol{F}_{A, H, K}(t)\right\|_{B(H)} \leq 1+t\left(2 \sum_{k=1}^{N}\left|a_{k}\right|+\|K\|_{L_{1}}\right), t \geq 0 .
$$

Operator-valued function $\boldsymbol{F}_{A, H, K}$ admits the representation

$$
\begin{aligned}
& \begin{array}{l}
\boldsymbol{F}_{A, H, K}(t)=\boldsymbol{F}_{0}(t)+t \sum_{k=1}^{N} a_{k}\left[S_{h_{k}} \boldsymbol{F}_{0}(t)+S_{-h_{k}} \boldsymbol{F}_{0}(t)\right] \\
+t t \int_{R} S_{y} \boldsymbol{F}_{0}(t) K(y) \mathrm{d} y
\end{array} \\
& \text { Since }\left\|\boldsymbol{F}_{0}(t)\right\|_{B(H)}=1 \text { then }
\end{aligned}
$$


$\left\|\boldsymbol{F}_{A, H, K}(t)\right\|_{B(H)} \leq 1+2 t|a|+t\|K\|_{L_{1}}$. Operator-valued function $\boldsymbol{F}_{0}(t), t>0$, is continuous in the strong operator topology and uniformly bounded in the norm topology, and the operators $\boldsymbol{S}_{ \pm h}$ is bounded and does not depend on the variable $t$. Therefore operator-valued function $\boldsymbol{F}_{A, H, K}(t)$ is continuous in the strong operator topology. According to lemmas 3 and 4 the function $\boldsymbol{F}_{A, H, K}(t), t \geq 0$, satisfies all conditions of Chernoff theorem. Hence the following statement is obtained.

Theorem 4. Let $K \in L_{1}(R)$. Then operator-valued function $\boldsymbol{F}_{A, H, K}(t), t \geq 0$, is equivalent by Chernoff to the semigroup $\boldsymbol{U}_{K}(t), t>0$.

\section{Remarks on Feynman-Kac Representation}

Using the result of Theorem 3, we obtain approximate solution of the Cauchy problem (1), (2) by sequence of multiple integrals, which integrand expression contains elementary functions and the initial condition. Therefore we obtain the solution by passing to the limit

$$
\begin{aligned}
& U(t) u_{0}(x) \\
& =\lim _{m \rightarrow \infty} \iint_{R R} \cdots \int_{R} F_{a, h}\left(t-t_{m-1}, x, y_{m-1}\right) F_{a, h}\left(t_{m-1}-t_{m-2}, y_{m-1}, y_{m-2}\right) \cdots F_{a, h}\left(t_{1}, y_{1}, y_{0}\right) u_{0}\left(y_{0}\right) \mathrm{d} y_{0} \cdots \mathrm{d} y_{m-1},
\end{aligned}
$$

where $t_{j}=\frac{j}{N} t$. For any $m \in \mathbf{N}$ the $m$-multiply integral under the limit operation defines the values of measure Feynman-Kac on cylindrical sets and hence, on the algebra of cylindrical sets in the space of continuous maps of semiaxis $R_{+}$into the coordinate space $R$ (see $[4,9])$.

Conversely we can obtaine the expression for the Cauchy problem solution by Feynman-Kac formula. The markovian measure $\mu$ (see $[10,11]$ ) is defined by means of semigroup $\boldsymbol{U}(t), t \geq 0$, on the algebra $\mathcal{A}$ of cylindrical sets in space $C\left(R_{+}, R\right)$ of continuous maps of semiaxis $[0,+\infty)$ into coordinate space $R$ by the following rule. The value of measure $\mu$ on an arbitrary cylindrical set $A=\left\{\xi \in C\left(R_{+}, R\right): \xi\left(t_{j}\right) \in B_{j}, j \in \overline{1, n}\right\}$ (where $B_{j}$ is bounded set from algebra $\mathcal{B}(R)$ of Borel subsets of $R, n=2,3, \cdots$ and $\left.0 \leq t_{1}<t_{2}<\cdots<t_{n}\right)$ is given by equality

$$
\begin{aligned}
& \mu(A) \\
& =\left(\chi_{B_{n}}, \boldsymbol{U}\left(t_{n}-t_{n-1}\right) \boldsymbol{P}_{B_{n-1}} \boldsymbol{U}\left(t_{n-1}-t_{n-2}\right) \cdots \boldsymbol{U}\left(t_{3}-t_{2}\right) \boldsymbol{P}_{B_{2}} \boldsymbol{U}\left(t_{2}-t_{1}\right) \chi_{B_{1}}\right)_{L_{2}(R)}
\end{aligned}
$$

(Here $\chi_{B}$-characteristic function of set $B$, and $\boldsymbol{P}_{B}$ - the projective operator of multiplication to characteristic function of set $B$ ).

Then according to the work [12] the following Feynman-Kac formula

$$
\begin{aligned}
& \left(\chi_{B}, u(t)\right)=\int_{C\left(R_{+}, R\right)} \chi_{B}(\xi(t)) u_{0}(\xi(0)) \mathrm{d} \mu(\chi), \\
& \forall t>0, B \in \mathcal{B}(R),
\end{aligned}
$$

uniquely defines the solution $u(t)=\boldsymbol{U}(t) u_{0}$ of the Cauchy problem (1), (2).

\section{Conclusion}

In this paper we obtain the approximation of solution of Cauchy problem for parabolic difference-differential equation with deviation of the space arguments by Feynman formulas. This result gives the opportunity to find the approximation of exact solution of Cauchy problems by using only $N$ integration with analitic function. Also this result gives the approximation of diffusion type random process with values in coordinate space such that the mean value of functional depending on this process is the solution of considered Cauchy problem.

\section{REFERENCES}

[1] A. D. Myshkis, "Mixed Functional Differential Equations," Journal of Mathematical Science, Vol. 129, No. 5, 2005, pp. 4111-4226. doi:10.1007/s10958-005-0345-2

[2] A. L. Skubachevski, "On Some Properties of Elliptic and Parabolic Functional-Differential Equations," Russian Mathematical Surveys, Vol. 51, No. 1, 1996, pp. 169-170. doi:10.1070/RM1996v051n01ABEH002765

[3] M. A. Vorontsov, Yu. D. Dumarevskii, D. V. Pruidze and V. I. Shmal'gauzen, "Izvestiya: The Academy of Sciences of the USSR," Atmospheric and Oceanic Physics, Vol. 52, No. 2, 1988.

[4] Y. A. Butko, R. L. Shilling and O. G. Smolyanov, "Feynman Formulae for Feller Semigroups," Doklady Mathematics, Vol. 82, No. 2, 2010, pp. 679-683. doi:10.1134/S1064562410050017

[5] A. L. Skubachevskii and R. V. Shamin "First Mixed Problem for a Parabolic Difference-Differential Equation," Mathematical Notes, Vol. 66, No. 1, 1999, pp. 113119. doi:10.1007/BF02674077

[6] O. G. Smolyanov and H. von Weizsacker, "Feynman 
Formulas Generated by Self-Adjoint Extensions of the Laplacian," Doklady Mathematics, Vol. 79, No. 3, 2009, pp. 335-338. doi:10.1134/S1064562409030090

[7] V. Zh. Sakbaev and O. G. Smolyanov, "Dynamics of a Quantum Particle with Discontinuous Position-Dependent Mass," Doklady Mathematics, Vol. 82, No. 1, 2010, pp. 630-633. doi:10.1134/S1064562410040332

[8] R. P. Chernoff, "Note on Product Formulas for Operator Semigroups," Journal of Functional Analysis, Vol. 2, No. 2, 1968, pp. 238-242. doi:10.1016/0022-1236(68)90020-7

[9] E. V. Dynkin, "Markovskie Processy," Fizmatgiz, Mos- cow, 1963.

[10] O. G. Smolyanov and E. T. Shavgulidze, “Kontinual'nye Integraly," MGU, Moscow, 1990.

[11] Yu. L. Daleckij and S. V. Fomin, "Mery i Differencial'nye Uravneniya v Beskonechnomernih Prostranstvah," Nauka, Moscow, 1983.

[12] V. Zh. Sakbaev and O. G. Smolyanov, "Diffusion and Quantum Dynamics of Particles with Position-Dependent Mass," Doklady Mathematics, Vol. 86, No. 1, 2012, pp. 460-463. 